\title{
СИНТЕЗ ИНФОРМАЦИОННЫХ МОДЕЛЕЙ ПРИНЯТИЯ РЕШЕНИЙ В СИСТЕМЕ УПРАВЛЕНИЯ ЭКОЛОГИЧЕСКОГО МОНИТОРИНГА
}

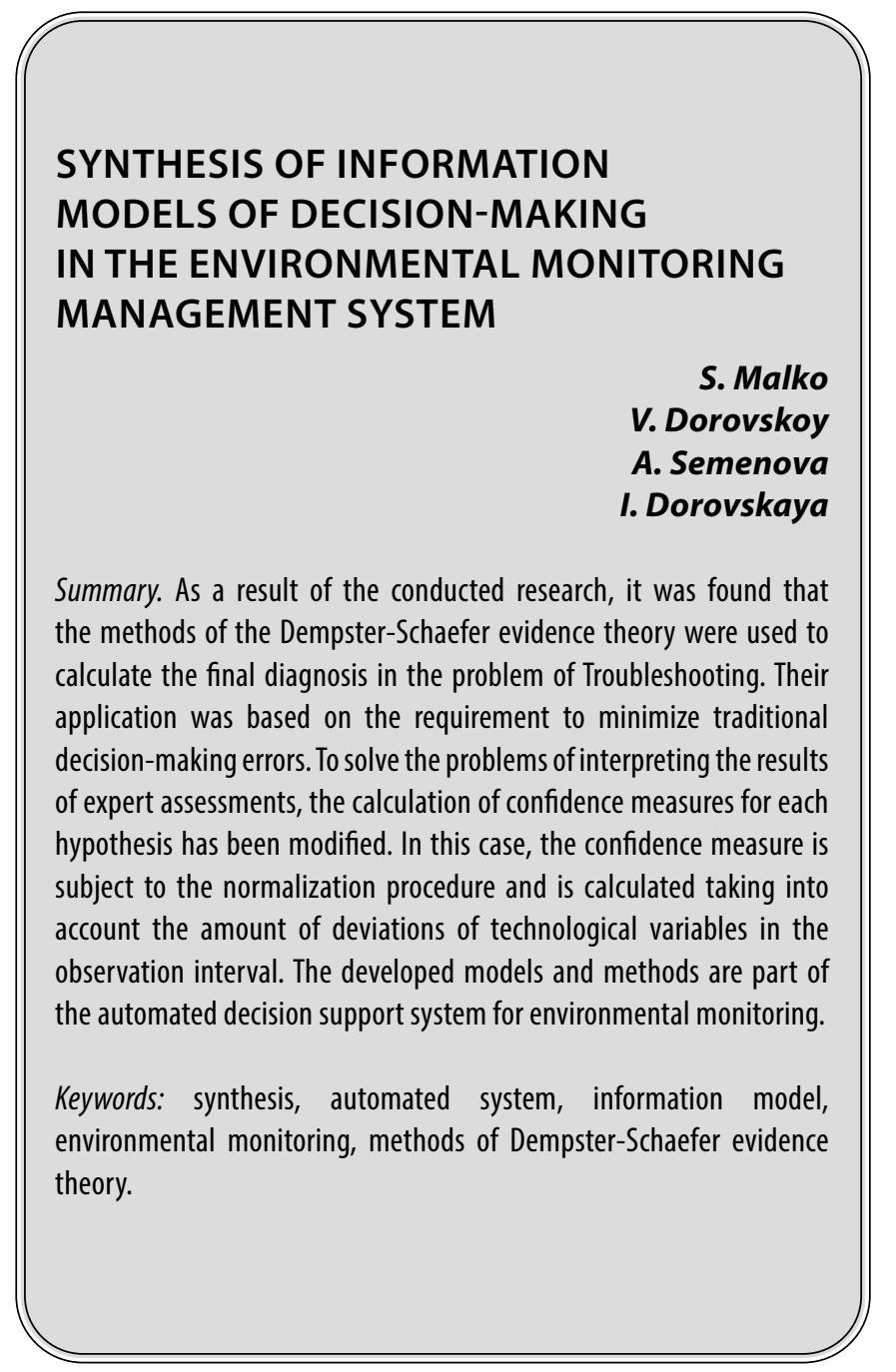

\section{Актуальность темы исслеАований.} Постановка проблемы

и нтенсивное воздействие человека на природу, негативные, часто необратимые последствия этого воздействия, обусловливают необходимость глубокого и всестороннего анализа проблемы взаимодействия общества и окружающей среды. Проблема улучшения экологической ситуации связана с необходимостью решения множества сложных нелинейных, трудно формализуемых задач, использующих, в первую очередь, результаты мониторинга окружающей среды. Разработка методов экологического мониторинга относится к числу фундаментальных научных исследований, объявленных в 1988 г. в Программе биосферных и эко-
Малько Сергей Владимирович

К.б.н., дочент, ФГБОУ ВО «Керченский государственный морской технологический университет», г. Керчь sergmalko@mail.ru

Доровской Владимир Алексеевич Д.Т.н., профессор, ФГБОУ ВО «Керченский государственный морской технологический университет», г. Керчь Семенова Анна Юрьевна

К.э.н., дочент, ФГБОУ ВО «Керченский государственный морской технологический университет», г. Керчь

Доровская Ирина Дмитриевна ФГБОУ ВО «Керченский государственный морской технологический университет», г. Керчь

Аннотация. В результате проведенных исследований установлено, что для вычисления окончательного диагноза в задаче поиска неисправностей применены методы теории свидетельств Демпстера-Шефера. Основанием их применения стало требование минимизации традиционных ошибок принятия решений. Для решения проблем интерпретации результатов экспертных оценок модифицирован расчет мер доверия для каждой гипотезы. При этом мера доверия подвергается процедуре нормализации и рассчитывается с учетом величины отклонений технологических переменных на интервале наблюдения. Разработанные модели и методы вошли в состав автоматизированной системы поддержки принятия решений экологического мониторинга.

Ключевые слова: синтез, автоматизированная система, информационная модель, экологический мониторинг, методы теории свидетельств Демпстера-Шефера.

логических исследований АН СССР на период до 2015 г. Необходимость решения задач поддержки принятия решений при оценке экологической обстановки связана, в частности с тем, что измерительные возможности реальных систем промышленно-экологической безопасности всегда ограничены. Эти ограничения касаются как плотности размещения контрольных пунктов, так и частоты выполняемых измерений, а значит оперативности получения требуемых данных. Сложное положение, связанное с концентрацией большого количества предприятий на сравнительно небольшой площади, определяет направленность работ как на осуществление производственного экологического контроля на предприятиях региона, так и контроля их совместного влияния на окружающую среду. 


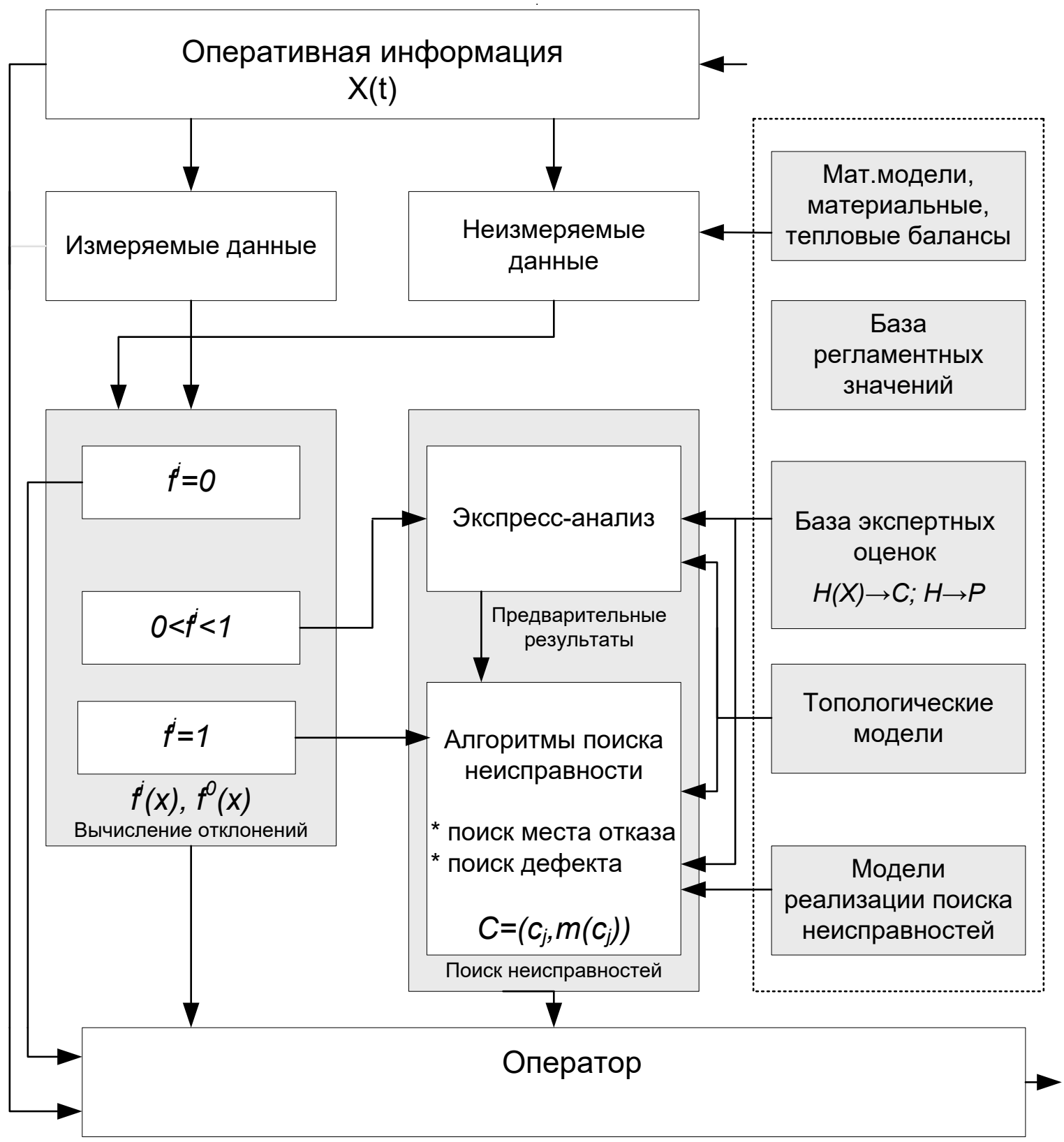

Рис. 1. Информационная модель автоматизированной системы поддержки принятия решений экологического мониторинга

\section{Цель работы}

Разработка информационных технологий поддержки принятия решений в региональной системе экологического мониторинга, позволяющих повысить уровень экологической и техногенной безопасности промышленного региона.

\section{Объект исслеАования}

Информационные технологии создания системы экологической и техногенной безопасности региона.

\section{Предмет исследования}

Модели и методы анализа, оценки и прогнозирования негативных последствий эксплуатации промышленных объектов.

\section{Метолы исслеАования}

Теоретической основой работы являются теория и методы принятия решений, математическая статистика и теория вероятностей, теория нечетких множеств. При разработке структуры информационной системы 


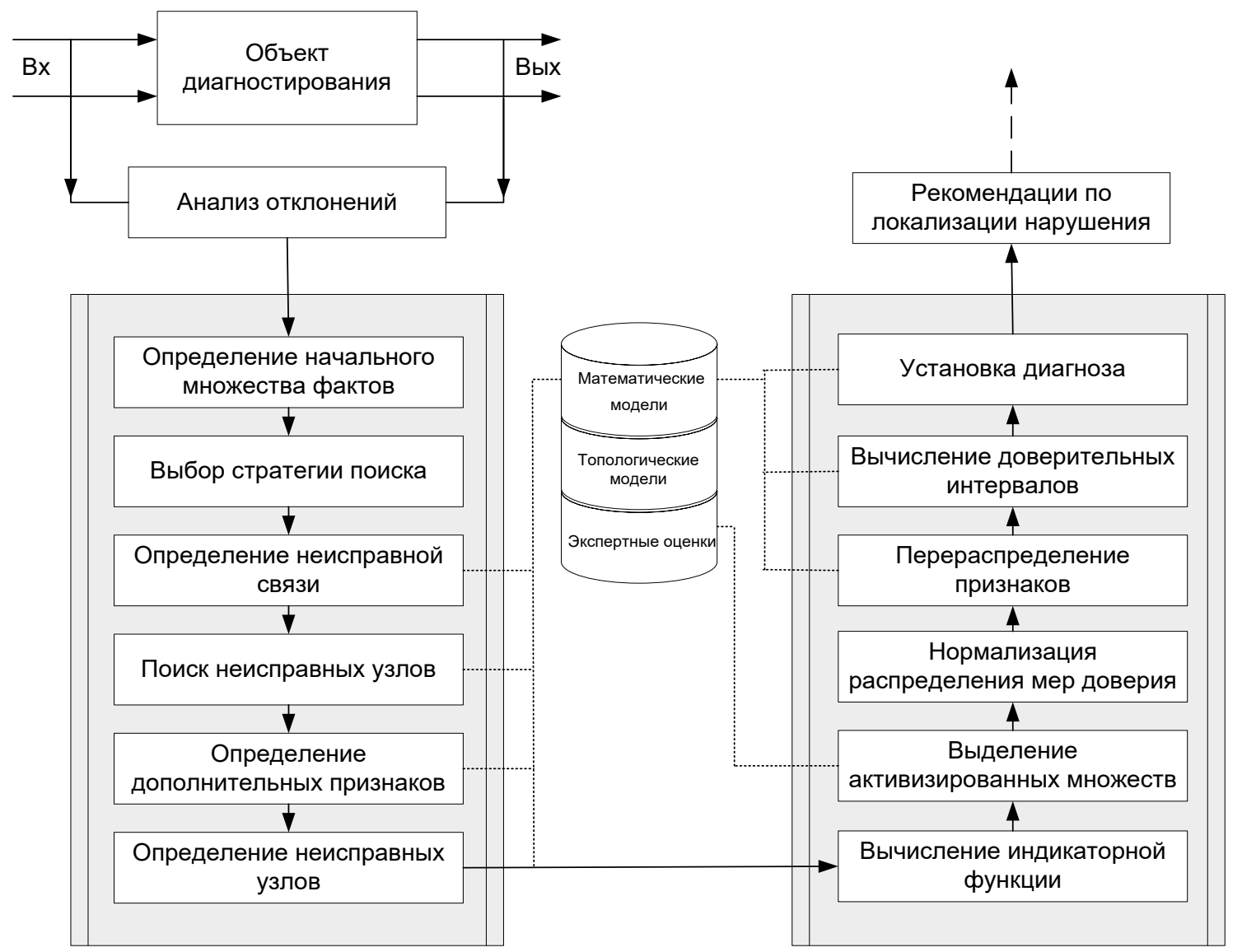

Рис. 2. Схема процесса поиска неисправностей и принятия решений

обеспечения приемлемого экологического риска использовались общие принципы создания информационных систем и средства системного анализа.

Моделирование и расчеты, связанные с анализом и количественной оценкой риска, проведены с использованием методов и средств вычислительной техники и технологий анализа данных в системах Mathcad, Maple, Statistica, Statgraphics Plus 5 и Ризекс.

\section{Результаты исслеАований}

Информационная модель автоматизированной системы, реализующей функции комплексного анализа химического производства с целью оперативного поиска неисправностей представлена на рисунке 1.

В состав модели включены:

- блок контроля параметров, главной задачей которого является предварительная обработка данных, формирование вспомогательных функций, учитывающих для каждой технологической переменной ее номинальные, критические и предельно допустимые значения;
- блок экспресс-анализа, выполняющий функции анализа ситуации и группировки информации с целью локализации постепенных отказов.

В случае, если состояние основных параметров выходят за рамки предельно допустимых включается система поиска неисправностей.

В соответствии с выбранной стратегией принятия решений в системе предполагается реализация двух возможных подходов:

1. поиск места отказа (определение части объекта, отказ которой вызвал неработоспособность подсистемы - неисправной технологической связи);

2. поиск дефекта (диагностирование, целью которого является определение места, причины и вида дефекта).

Общая схема процесса поиска неисправностей состоит из следующих этапов (рисунок 2):

1. Определение начального множества факторов, соответствующее текущему состоянию.

2. Регистрация нарушения диагностической переменной (непосредственное измерение, наруше- 
Таблица 1. Фрагмент результатов экспертной оценки для производства формальдегида

\begin{tabular}{|l|l|l|l|l|l|} 
Наименование диагностической переменной & \multicolumn{3}{|l}{ Технологические связи } \\
\cline { 2 - 6 } & $\mathbf{c}_{1}$ & $\mathbf{c}_{2}$ & $\mathbf{c}_{3}$ & $\mathbf{c}_{4}$ & $\mathbf{c}_{5}$ \\
\hline Объемный расход метанола & 0,5 & 0,3 & & \\
\hline Объемный расход демводы & 0,4 & 0,1 & 0,1 & & 0,1 \\
\hline Температура под сетками & 0,4 & 0,1 & 0,1 & 0,2 & 0,1 \\
\hline Температура подконтактных газов & 0,3 & 0,4 & 0,1 & & \\
\hline Массовая доля кислорода & 0,1 & 0,5 & 0,4 & & \\
\hline Температура после п. 21 & & 0,3 & & 0,2 & 0,2 \\
\hline Температура над 5 тарелкой & 0,2 & & 0,1 & 0,1 & \\
\hline
\end{tabular}

Таблица 2. Множества, активизированные появлением отклонений

\begin{tabular}{|c|c|c|c|c|c|c|}
\hline \multirow{3}{*}{ Наименование диагностической переменной } & \multirow{3}{*}{$\begin{array}{l}\text { Диапазон нормальной } \\
\text { работоспособности } \\
{\left[\mathbf{d}_{\min } ; \mathbf{d}^{\max }\right]}\end{array}$} & \multicolumn{5}{|c|}{ Технологические связи } \\
\hline & & \multicolumn{5}{|c|}{$\boldsymbol{\mu}_{i}\left(\mathbf{c}_{j}\right)$} \\
\hline & & $\mathrm{c}_{1}$ & $c_{2}$ & $c_{3}$ & $\mathbf{C}_{4}$ & $\mathbf{c}_{5}$ \\
\hline Температура под сетками $\left(H_{8}\right)$ & $620-780^{\circ} \mathrm{C}$ & 0,3 & 0,4 & 0,1 & & \\
\hline Массовая доля кислорода $\left(H_{21}\right)$ & $0,24-0,40$ & & 0,3 & & 0,2 & 0,2 \\
\hline
\end{tabular}

ние выходных характеристик математических моделей, нарушение балансовых соотношений).

3. Процедура поиска неисправностей:

- определение начального множества фактов неисправностей и вероятности наличия источника по каждой технологической связи;

- определение неисправной технологической подсистемы (связи), для которой наиболее вероятно наличие в ее составе отказавшего элемента;

- сужение области поиска, определение неисправного узла (нескольких узлов) с указанием распределения степеней уверенности в наличии дефекта по множеству неисправных связей; процедура принятия решения о наличии неисправной связи.

4. Выдача результатов диагноза и рекомендаций по локализации нарушения.

Ниже представлен пример совместного использования представленных в данном разделе моделей для технологического процесса производства формалина.

Пусть в результате прямых замеров и расчетов по диагностической модели зарегистрировано 2 события:

1. увеличение температуры в контактном аппарате сверх нормативной (переменная $H_{8},\left(f\left(H_{8}\right)\right)$ );

2. увеличение массовой доли кислорода (переменная $H_{21}\left(f\left(H_{21}\right)\right)$ ).
При этом активизируется нечеткое множество возможно неисправных связей $C_{i}=\left\{\left(c_{j} ; \mu_{i}\left(c_{i}\right)\right)\right\}$, где $\mu_{i}\left(c_{i}\right)$ - степень принадлежности элемента $c_{j}$ множеству $C_{i}$ (степень уверенности эксперта в наличии источника нарушений).

Шаг 1. Вычисление индикаторной функции.

Значение переменной $\mathrm{H}_{8}$ на интервале наблюдений заключено в пределах: $H_{8}=[780 \ldots 828]$, Регламентные значения $-\left[d_{\min }, d^{\max }\right]=[620 \ldots 780] . \Delta=160$. В соответствии с (1)

$$
|X|: X=\left\{x(t) \mid x(t) \in\left[d_{\min }, d^{\max }\right]\right\}
$$

где $x(t)$ - текущее значение диагностической переменной; $\left[\mathrm{d}_{\min }, \mathrm{d}^{\mathrm{max}}\right]$ - интервал допустимых значений параметра $t$. величина отклонения диагностической переменной вычисляется по максимальному зафиксированному значению:

$$
f\left(H_{8}\right)=(780-828+80) /(160)=0,2 ;
$$

Расчетное значение переменной $\mathrm{H}_{2 I}=[0,40 \ldots 0,47]$ при заданном диапазоне $\left[d_{m i n}, d^{\max }\right]=[0,24 \ldots 0,40]$. Тогда, в соответствии с (1) $\mathrm{f}\left(\mathrm{H}_{21}\right)=0,56$.

Из табл. 1 определяются активизированные множества (табл. 2.). 
Таблица 3. Результаты расчетов

\begin{tabular}{|l|l|l|l|l|l|l|l|l|}
\hline $\mathbf{C}_{i}$ & $\mathbf{c}_{1}$ & $\mathbf{c}_{2}$ & $\mathbf{c}_{3}$ & $\mathbf{c}_{4}$ & $\mathbf{c}_{5}$ & $\mathbf{c}_{4}, \mathbf{c}_{5}$ & $\mathbf{c}_{0}$ \\
\hline $\mathrm{m}(\mathrm{Ci})$ & 0,03 & 0,37 & 0,01 & 0 & 0 & 0,02 & 0,39 \\
\hline $\mathrm{Bel}(\mathrm{Ci})$ & 0,03 & 0,37 & 0,01 & 0 & 0 & 0,02 & 0,39 \\
\hline $\mathrm{PI}(\mathrm{Ci})$ & 0,42 & 0,76 & 0,4 & 0,39 & 0,39 & 0,59 & 1,0 \\
\hline
\end{tabular}

$$
\begin{aligned}
& \mathrm{m}\left(\mathrm{H}_{8}\right)=\left\{\left(\mathrm{c}_{1} ; 0,3\right),\left(\mathrm{c}_{2} ; 0,4\right),\left(\mathrm{c}_{3} ; 0,1\right)\right\} ; \\
& \mathrm{m}\left(\mathrm{H}_{21}\right)=\left\{\left(\mathrm{c}_{2} ; 0,3\right),\left(\mathrm{c}_{4} ; 0,2\right),\left(\mathrm{c}_{5} ; 0,2\right)\right\} .
\end{aligned}
$$

Шаг 2. Нормализация мер доверия

Нормализованная мера достоверности событий определяется как (2):

$$
m_{l}\left(C^{f}\right)=\frac{\mu\left(C_{i}\right)}{\sum_{C_{i} \subseteq C^{f}} \mu\left(C_{i}\right)}
$$

где $C_{i}$ - любое событие из множества $C_{f}$, состоящее в неисправности одной или нескольких технологических связей.

$$
\begin{aligned}
& \mathrm{m}\left(\mathrm{H}_{8}\right)=\left\{\left(\mathrm{c}_{1} ; 0,37\right),\left(\mathrm{c}_{2} ; 0,5\right),\left(\mathrm{c}_{3} ; 0,12\right)\right\} ; \\
& \mathrm{m}\left(\mathrm{H}_{21}\right)=\left\{\left(\mathrm{c}_{4} \mathrm{c}_{5} ; 0,4\right),\left(\mathrm{c}_{2} ; 0,6\right)\right\} .
\end{aligned}
$$

Шаг 3. Перераспределение мер доверия проводится с использованием выражения (3):

$$
m_{i}^{n}\left(C^{f}\right)=f^{i}\left(x_{i}\right) \cdot \frac{\mu\left(C_{i}\right)}{\sum_{C_{i} \subseteq C^{f}} \mu\left(C_{i}\right)}
$$

где $f^{i}(x)$ определяется по (1) при этом $c_{o}$ рассчитывается как оценка правдоподобия.

$$
\begin{aligned}
& m^{\prime}\left(H_{8}\right)=\left\{\left(c_{1} ; 0,07\right),\left(c_{2} ; 0,1\right),\left(c_{3} ; 0,02\right),\left(c_{o} ; 0,81\right)\right\} ; \\
& m^{\prime}(\mathrm{H} 21)=\left\{\left(c_{4} \vee c_{5} ; 0,22\right),\left(c_{2} ; 0,34\right),\left(c_{o} ; 0,44\right)\right\} .
\end{aligned}
$$

Определение меры конфликта проводится по формуле (4).

Для объединения различных свидетельств с распределениями мер доверия $m_{1}$ и $m_{2}$ в пользу одной гипоте-

\begin{tabular}{|c|c|c|c|}
\hline$m_{m^{\prime}\left(\mathrm{H}_{21}\right)} m^{\prime}\left(\mathrm{H}_{8}\right)$ & $\begin{array}{l}c_{1} \\
0,07\end{array}$ & $\begin{array}{l}c_{2} \\
0,1\end{array}$ & $\begin{array}{l}c_{3} \\
0,02\end{array}$ \\
\hline $\mathrm{C}_{4} \mathrm{~V}_{\mathrm{C}_{5}}$ & $\begin{array}{l}\varnothing \\
0,015\end{array}$ & \begin{tabular}{l|l} 
\\
0,02
\end{tabular} & \begin{tabular}{|l|}
$\varnothing$ \\
0,004
\end{tabular} \\
\hline \begin{tabular}{|l|}
$C_{2}$ \\
0,34
\end{tabular} & $\begin{array}{l}\varnothing \\
0,02\end{array}$ & $\begin{array}{l}c_{2} \\
0,03\end{array}$ & $\begin{array}{l}\varnothing \\
0,006\end{array}$ \\
\hline
\end{tabular}
зы при $m_{1,2}(\varnothing)=0$, используется правило Демпстера-Шефера:

$$
m_{1,2}\left(C^{f}\right)=\frac{1}{1-\mathrm{K}} \sum_{C_{i} \cap C_{j}=C \neq 0} m_{1}\left(C_{i}\right) m_{2}\left(C_{j}\right)
$$

где $K$ - мера конфликта ( $m(\varnothing)$ - мера доверия, приходящаяся на пустое множество) вычисляется по формуле (5): $\mathrm{m}(\varnothing)=0,02+0,02+0,006+0,015+0,004=0,1$.

Расчет мер доверия, учитывающих обе гипотезы о неисправности связей, проводится согласно (3):

$$
\begin{aligned}
& \mathrm{m}\left(\mathrm{c}_{1}\right)=\left(0,07^{*} 0,44\right) / 0,9=0,03 ; \\
& \mathrm{m}\left(\mathrm{c}_{2}\right)=(0,03+0,04+0,27) / 0,9=0,37 ; \\
& \mathrm{m}\left(\mathrm{c}_{3}\right)=(0,01) / 0,9=0,01 ; \\
& \mathrm{m}\left(\mathrm{c}_{4} \vee \mathrm{c}_{5}\right)=(0,18) / 0,9=0,2 ; \\
& \mathrm{m}\left(\mathrm{c}_{o}\right)=0,36 / 0,9=0,39 .
\end{aligned}
$$

Результирующее распределение вероятностей:

$$
\mathrm{m}\left(\mathrm{H}_{8}, \mathrm{H}_{21}\right)=\left\{\left(\mathrm{c}_{1} ; 0,03\right),\left(\mathrm{c}_{2} ; 0,37\right),\left(\mathrm{c}_{3} ; 0,01\right),\left(\mathrm{c}_{4} \vee \mathrm{c}_{5} ; 0,2\right),\right.
$$
$\left.\left(c_{o} ; 0,39\right)\right\}$;

Шаг 4. Определение соответствующих интервалов и определение связи, содержащей неисправный элемент. Результаты расчетов представлены в табл.3.

Таким образом, в результате проведенных расчетов, при заданном пороговом уровне доверия, основанный на теории свидетельств Демпстера-Шефера анализ технологической ситуации производства формалина, при которой зарегистрировано повышение температуры выше допустимого и увеличение массовой доли кислорода, приводит к следующему распределению диагностических оценок:

$$
\mathrm{c}_{l}[0,03 ; 0,42], \quad \mathrm{c}_{2}[0,37 ; 0,76], \quad \mathrm{c}_{4}[0 ; 0,59], \quad \mathrm{c}_{5}[0 ; 0,59],
$$
$\mathrm{C}[1,0 ; 1,0]$

Ввиду того, что по определению меру доверия $(\mathrm{Bel})$ называют нижней вероятностью, а меру правдоподобия $(P l)$ соответственно верхней вероятностью возможна следующая интерпретация результирующего 


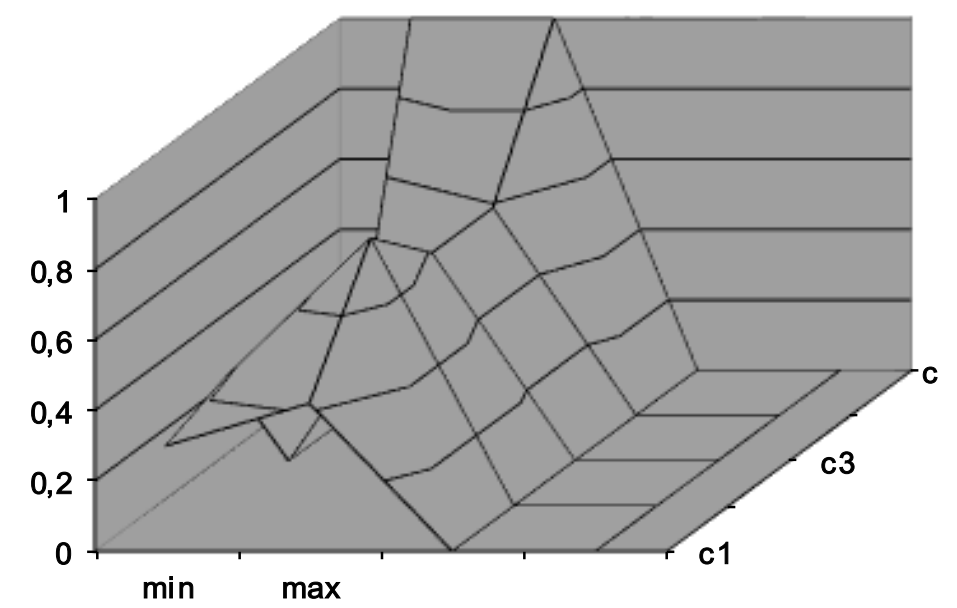

Рис. 3. Распределение вероятностей диагноза, вычисленного с использованием методов теории свидетельств Демпстера-Шефера

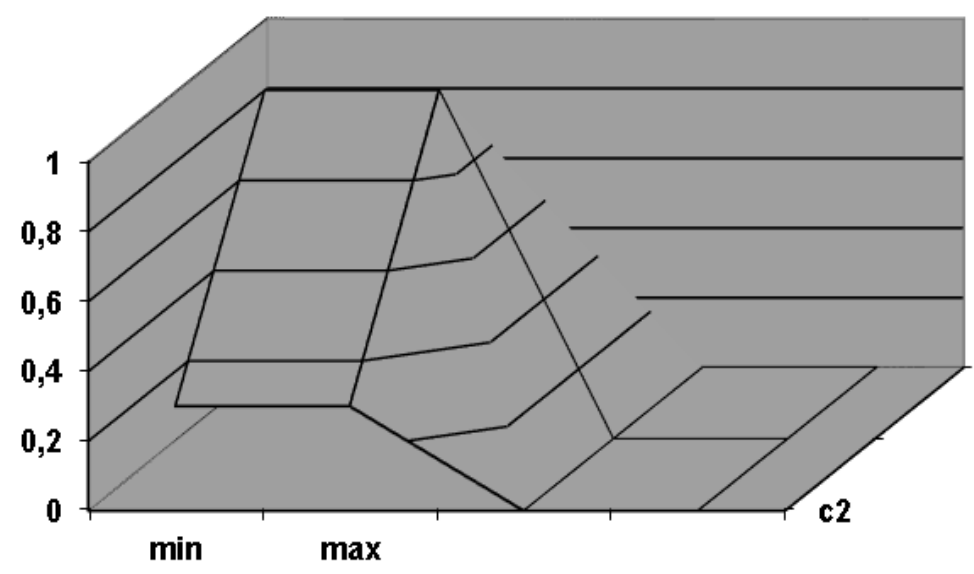

Рис. 4. Распределение вероятностей диагноза с использованием принципа обобщения

диагноза: вероятность наличия неполадок в связи $c_{2}$ не менее $37 \%$ и не более $76 \%$. По результатам расчетов, гипотезы о наличии неполадок в связях $c_{1}, c_{3}, c_{4}$, $c_{5}$ не подтвердились. Даже при общем подходе к задаче экспертной классификации применение правила Демпстера-Шефера позволяет сделать полученные данные не противоречивыми и использовать их для объединения с результатами математического моделирования. Так как на настоящий момент не существует законченной теории нечетких случайных событий, использование комбинированного подхода с позиций теории нечетких множеств и Демпстера-Шефера вполне оправдано.

На рис. 3, 4 представлены результаты вычислений экспертных оценок соответственно по методу Демпстера-Шефера и с помощью принципа обобщения. По полученным экспериментальным данным установлено, что результирующий диагноз с использованием принципа обобщения определяет возможность неполадки в газовой связи подачи воздуха на смешение, игнорируя при этом гипотезу о наличии неполадок в гидравлической связи подачи метанола.

\section{Эак^ючение}

В результате проведенного синтеза установлено: для вычисления окончательного диагноза в задаче поиска неисправностей применены методы теории свидетельств Демпстера-Шефера. Основанием их применения стало требование минимизации традиционных ошибок принятия решений. Для решения проблем интерпретации результатов экспертных оценок модифицирован расчет мер доверия для каждой гипотезы. При этом мера доверия подвергается процедуре нормализации и рассчитывается с учетом величины отклонений технологических переменных на интервале наблюдения. Разработанные модели и методы вошли в состав автоматизированной системы поддержки принятия решений экологического мониторинга. 


\section{ЛИТЕРАТУРА}

1. Абрамов Ю.Ф. Региональная безопасность (эколого-информационный аспект) / Абрамов Ю.Ф., Душутин В.К., Сидоркина Е.В.— Иркутск: Изд-во Иркутского госуниверситета. 2003. - 200 c.

2. Абрамович Ф.П. Решение нечетких систем линейных алгебраических уравнений LR-типа / Абрамович Ф.П., Вагенкнехт М.А., Хургин Я.И. // Методы и системы принятия решений. - Рига: РПИ, 1987.—- С. 35-47.

3. Александреску А. Современное проектирование на C++ / Александреску Андрей.—Cерия C++ In-Depth, т. 3.: Пер. с англ. — М.: Издательский дом «Вильямс», 2002.- 336 с.

4. Белов П.Г. Системный анализ и моделирование опасных процессов в техносфере / Белов П.Г.— М.: Академия, 2003. — 512 с.

5. Варзар Р.Л. Разработка автоматизированной системы мониторинга загрязнения атмосферного воздуха: [Электронный ресурс] / Варзар Р.Л.— Режим доступа: http://masters.donntu.edu.ua/2009/fvti/varzar/diss/index.htm.

6. Владимиров В.А. Оценка риска и управление техногенной безопасностью. Монография. / Владимиров В.А., Измалков В.И., Измалков А.В.- М.: ФИД «Деловой экспресс» - 2002-184 с.

7. Кривогуз Д.О. Влияние склоновых процессов на состояние землепользования Керченского полуострова / Д.0. Кривогуз // Сборник материалов I Всероссийской научно-практической конференции «Техносферная безопасность. Современные реалии».—- Махачкала: Дагестанский государственный технический университет, 2019. - С. 96-100

8. Теория выбора и принятия решений: Учебное пособие.— М.: Наука. Главная редакция физико-математической литературы, 1982.

(c) Малько Сергей Владимирович ( sergmalko@mail.ru ), Доровской Владимир Алексеевич,

Семенова Анна Юрьевна, Доровская Ирина Дмитриевна.

Журнал «Современная наука: актуальные проблемы теории и практики»

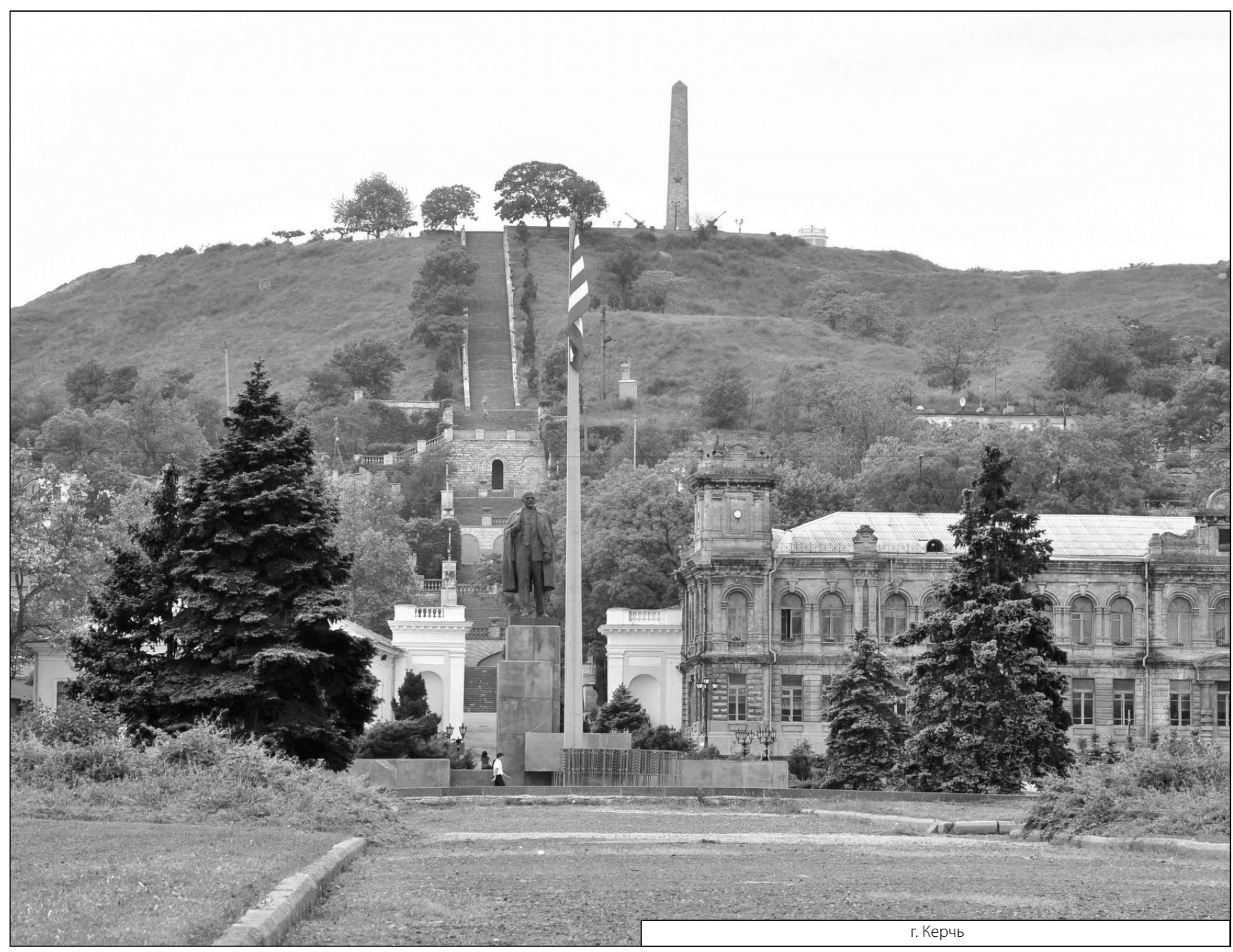

